\title{
Liquid Chromatography under Limiting Conditions of Desorption IV. Separation of Macromolecules According to Their Stereoregularity
}

\author{
By Dusan BEREK,${ }^{1, *}$ Tatsuki KITAYAMA, ${ }^{2}$ Koichi HATADA, ${ }^{2}$ Hirotaka IHARA,${ }^{3}$ \\ Ignac CAPEK, ${ }^{1}$ and Eberhard BORSIG ${ }^{1}$
}

\begin{abstract}
Liquid chromatography under limiting conditions of adsorption (LC LCD) is a novel technique for separation of polymers that exhibit different extent of adsorption on the porous column packing of appropriate polarity. LC LCD is based on the selective "barrier effect" induced by a narrow zone of the adsorption promoting liquid that is introduced into column in front of separated macromolecules of different nature. The small molecules that form the barrier elute slowly because they permeate the packing pores. The partially or fully pore excluded polymer molecules elute rapidly. The barrier hinders fast elution of macromolecules that tend to be adsorbed within column packing; their elution is slowed down. At the same time, the non-adsorbed polymer species elute non-hindered. It is shown that due to the controlled barrier effect even a very small difference in the extent of adsorption between isotactic and syndiotactic macromolecules of poly(methacrylate)s can lead to the significant distinction in their retention volumes. While the direct LC LCD separation of poly(methyl methacrylate)s of different stereoregularity is often precluded by the aggregation of unlike macromolecules, the highly stereoregular poly(ethyl methacrylate)s of distinct kind can be easily discriminated irrespective of their molar mass. Simultaneously, the low molecular and oligomeric impurities can be removed and, if necessary they can be identified and quantified by independent methods. In this way, LC LCD can assist purification of stereoregular polymers and serve as the first step in the twodimensional liquid chromatography of their non-aggregated mixtures.

KEY WORDS: Stereoregular Polymers / Separation / Liquid Chromatography / Limiting Conditions of Adsorption /
\end{abstract}

Separation of chemically identical synthetic macromolecules according to architecture of their molecules represents a challenge from both scientific and technological points of view because physical and utility properties of some polymeric materials largely depend on this characteristic. Stereoregularity of polymers represents a typical example that demonstrates the substantial role of molecular architecture, while poly(methacrylate)s, polystyrene and polypropylene represent the technologically important representatives of this group.

The prerequisite for comprehensive molecular characterization of synthetic polymers is their separation according to the parameter under study. For the linear homopolymers of given nature, the decisive molecular characteristic is their molar mass both its average and distribution. However, one deals with multiple distributions of the molecular characteristics in the case of complex polymers such as copolymers, polymer blends, and also stereoregular polymers, because not only their molar mass but also their chemical structure and/or physical architecture exhibits a degree of non-uniformity.

Presently the most important method of molecular characterization of synthetic polymers is size exclusion chromatography, SEC called also gel permeation chromatography, GPC. As known, retention of macromolecules in SEC/GPC is largely controlled by the changes of their conformational entropy while permeating pores of the column packing. Consequently,
SEC separates macromolecules according to their size in solution. As this size simultaneously depends on all above molecular characteristics, it is evident that SEC alone cannot produce exact, quantitative information on the molar mass of complex polymers. Moreover, the separation selectivity of SEC is generally rather limited, and this does not allow discrimination of simple homopolymers that possess similar or even identical molar mass. To separate complex polymers, additional retention mechanism(s) are to be added; these are driven by the enthalpic interactions within chromatographic column.

It was shown that the extent of adsorption of macromolecules on a given surface is affected not only by their chemical structure, composition but also by their molar mass and by their physical architecture including their stereoregularity. ${ }^{1-3}$ This allowed application of eluent gradient liquid chromatography, EG LC, ${ }^{4-9}$ and liquid chromatography under critical conditions of enthalpic interactions, $\mathrm{LC} \mathrm{CC}^{10-12}$ for selective separation of macromolecules of different nature (for review, see for example ${ }^{13,14}$ ). In EG LC the mobile phase composition is changed in a controlled way. Macromolecules that exhibit different interactivity with the column packing are eluted at distinct eluent compositions, within different retention volumes. In contrast, LC CC is an isocratic procedure. Under critical conditions, the exclusion and enthalpic interactions for certain kind of macromolecules mutually compensate so that

\footnotetext{
${ }^{1}$ Polymer Institute, Slovak Academy of Sciences, 84236 Bratislava, Slovakia

2 Department of Chemistry, Osaka University, Osaka 560-8531, Japan

${ }^{3}$ Department of Applied Chemistry and Biochemistry, Kumamoto University, Kurokami, Kumamoto 860-8555, Japan

*To whom correspondence should be addressed (E-mail: dusan.berek@savba.sk).
} 
their retention volumes do not depend on their molar mass. Other kinds of macromolecules are eluted under (prevailingly) exclusion or interaction conditions to be separated from the "critically" eluted species. Both EG LC ${ }^{7}$ and LC CC ${ }^{15-18}$ were also applied to mutual separation of macromolecules differing in their stereoregularity. A series of highly stereoregular narrow molar mass distribution isotactic, it.-, and syndiotactic, st.-poly(methacrylate)s, separately injected into the EG LC or LC CC column eluted in different retention volumes even if molar masses were almost identical. Adsorption retention mechanism was applied. These results indicated a possibility to discriminate mixtures of macromolecules according to their stereoregularity. However, due to the mutual aggregation of high molecular it.- and st.-macromolecules of PMMA in solution, ${ }^{16}$ their mixtures produced ill shaped peaks in the ordinary LC CC solvent mixtures. The aggregation suppressing chloroform was also applied. ${ }^{16} \mathrm{CHCl}_{3}$ is a relatively weak solvent considering bare silica gel. It only slightly promotes adsorption of PMMA and the critical adsorption point has been already reached by addition of a rather small amount of a strong solvent, ethanol. Consequently, only a narrow eluent composition range could be applied. Moreover, $\mathrm{CHCl}_{3}$ is difficult to work with because its repeatable purification is rather problematic. On the other hand, the mixtures of the narrow molar mass distributed non-aggregating poly(ethyl methacrylate)s, PEMA of similar molar mass but of distinct tacticity were base line separated in the LC CC columns applying mixtures of usual solvents such as tetrahydrofuran and hexan. ${ }^{17,18}$ Eventually, fractions of PEMA produced by SEC were on line separated according to their streoregularity with help of LC CC. Unfortunately, LC CC suffers from several drawbacks, such as

-high sensitivity of many LC CC chromatographic systems toward small variations of experimental conditions, especially to fluctuations in the eluent composition, pressure, and temperature, as well as to changes in the column interactivity ${ }^{19,20}$

-extensive broadening of the chromatographic zones ${ }^{19-21}$

-reduced recovery of polymers with increased molar mass; this makes LC CC hardly applicable to molar masses over $100 \mathrm{~kg} \cdot \mathrm{mol}^{-1} \cdot{ }^{21-25}$ The latter shortage may also bias the EG LC separations. $^{23,26}$

Cho et al. ${ }^{27}$ attempted to separate PEMAs according to their stereoregularity with help of temperature gradient interaction chromatography, TGIC. TGIC is based on the combined effect of the exclusion and the enthalpic interactions of the macromolecules, while the extent of enthalpic interaction is controlled by temperature variations. ${ }^{28,29}$ TGIC is known for its high resolution of macromolecules in term of their molar mass. By an irony of fate, the latter feature proved to be the issue for the application of TGIC to stereoregular polymers since two molecular characteristics, namely molar mass and stereoregularity of macromolecules simultaneously affected retention volumes of samples. Moreover, TGIC may suffer from the problems with the exact control of the column temperature, as well as from the reduced sample recovery.
Some of the above shortages of EGLC, LC CC and TGIC can be avoided or at least mitigated by the application of liquid chromatography under limiting conditions of enthalpic interactions, LC LC. ${ }^{14,30,31}$ In LC LC, the exclusion and interaction separation mechanisms are combined in an unconventional way. The difference is utilized in elution rate of small molecules and macromolecules within the liquid chromatographic column packed with porous particles. Small molecules of eluent permeate practically all pores of the column packing and therefore their elution rate is low. On the contrary, the partially or fully pore excluded macromolecules are transported along the column much faster. Appropriately chosen small molecules promote enthalpic interactions such as adsorption, enthalpic partition or precipitation of the relevant polymeric sample constituents. The above interactions result in the additional retention mechanism, which may decelerate elution of macromolecules. If introduced into an LC LC column before the polymeric sample, interaction promoting small molecules may create a slowly eluting barrier, which hinders fast elution of the interacting macromolecules. Under identical experimental conditions, the non-interacting polymer species elute freely in the SEC mode. In this way, the interacting macromolecules are easily separated from the non-interacting ones. The LC LC method that applies adsorption retention mechanism induced by a narrow zone of adsorption promoting liquid, an adsorli that precedes sample solution is denoted liquid chromatography under limiting conditions of desorption, LC LCD. ${ }^{30,31}$ LC LCD was used in the separation of minor $(<1 \%)$ macromolecular admixtures from the major constituents of polymer blends including parent homopolymers from the diblock copolymers. $^{32-34}$ The method was shown to exhibit low dependance from the eluent composition, which provides its increased experimental feasibility. Further, the sample recovery of LC LCD was very high or even complete and the narrow, focused peaks were produced. ${ }^{35}$ All these features qualify LC LCD as suitable partner for the advanced twodimensional liquid chromatographic methods of comprehensive polymer separation and molecular characterization. ${ }^{36,37}$ High selectivity of the LC LCD separations was attained when the difference was relatively large in adsorptivity of unlike polymer chains in model polymer blends and in block copolymers that contained parent homopolymers. ${ }^{32-34}$ It was of interest to evaluate the LC LCD behavior of such macromolecules that exhibit only a minute difference in their adsorptivity and to attempt extension of the method applicability also to separation of polymers according to their tacticity. The selected highly stereoregular poly(methyl methacrylate)s and poly(ethyl methacrylate)s served as appropriate models.

\section{EXPERIMENTAL}

\section{Materials}

The narrow molar mass distributed isotactic (it.-) and syndiotactic (st.-) poly(methyl methacrylate)s, PMMAs and poly(ethyl methacrylate)s, PEMAs were applied. They exhib- 
ited high degree of stereoregularity. Samples were prepared by anionic polymerization: it.-PMMA, ${ }^{38,39}$ it.-PMMA, ${ }^{40,41}$ it.-PEMA, ${ }^{42}$ and st.-PEMA. ${ }^{43}$ Their weight average molar mass values ranged from 12.3 to $157 \mathrm{~g} \cdot \mathrm{mol}^{-1}$ for PMMAs and from 4.9 to $56.2 \mathrm{~kg} \cdot \mathrm{mol}^{-1}$ for PEMAs. The detailed molecular characteristics of both polymer groups are given in papers. ${ }^{15-17}$ Polystyrene, PS "standards" with molar mass averages ranging from 0.8 to $498 \mathrm{~kg} \cdot \mathrm{mol}^{-1}$ with narrow molar mass distribution were from Pressure Co., USA.

The columns $7.5 \times 300 \mathrm{~mm}$ were packed with bare silica gels Kromasil 60, and Kromasil 100 from Eka Chemicals, Sweden with effective pore sizes 6 and $10 \mathrm{~nm}$, respectively. With exception of only one set of experiments, Kromasil 60 was applied in the measurements. The use of Kromasil 100 is explicitly specified in the text and in the corresponding Figure caption.

Tetrahydrofuran, THF, and toluene were applied as eluent and barrier components while DMF served as a strong displacer. Both THF and toluene are thermodynamically very good solvents for PS, PMMA and PEMA. ${ }^{44}$ The co-nonsolvency effects are improbable and therefore the danger of mixed retention mechanism adsorption/precipitation is low. Analytical grade both THF, and toluene were from CentralChem, Slovakia, and DMF of HPLC grade was from Scharlau, Spain. THF and toluene were distilled immediately before use. THF was stabilized with $0.2 \mathrm{~g} \cdot \mathrm{L}^{-1}$ of 2,6-di-tercbutyl-4-methyl phenol. The model admixtures were tricresyl phosphate, TCP from Slavus, Slovakia and poly(propylene oxide), PPO with effective molar mass $0.4 \mathrm{~kg} \cdot \mathrm{mol}^{-1}$ from Union Carbide, USA.

In our previous studies, ${ }^{33}$ differences were observed in the LC LCD retention of identical samples applying the same column packings, eluent and barrier in dependence on THF used. The blocking efficiency of barriers depended on supplier of THF though the solvent grades proclaimed were "identical." It is likely that traces of impurities present in THF, for example water or degradation products, strongly affected the chromatographic strength of the solvent. Therefore the entire sets of measurements have always been performed with the same stock of stabilized and well closed THF kept in dark.

\section{Measurements}

The apparatus used, as well as the arrangement of experiments were described in detail in recent publication. ${ }^{33}$ Therefore, only the basic information are given here.

The pump was from Watrex, Czech Republic, and the evaporative light scattering detector, ELSD, was from Polymer Laboratories, UK. The data supplied by detector were collected and processed with the software Baseline from Waters, USA or WinGPC from Polymer Standards Service, Germany. In dependence on the amount of sample introduced into column, the software automatically adjusted the peak sizes. This electronic manipulation was especially pronounced if the samples were spiked with admixtures. The flow rate was $1 \mathrm{~mL} \cdot \mathrm{min}^{-1}$. The water-jacketed column oven from Chroma,
Austria was kept at $30 \pm 0.1^{\circ} \mathrm{C}$. Eluent was pre-thermostated within a two meter long $0.7 \mathrm{~mm}$ I.D. capillary situated in the column oven. A tandem of three injection valves from Rheodyne, USA, was applied. One of them served for introduction of sample into the LC LCD column. It was followed by the second valve, which enabled the independent injection of the barrier(s) into the system before the sample. This valve was switched into its starting (loop filling) position after the barrier has been injected and before sample was introduced. This means that the injected solution did not flow through the barrier loop. In most experiments, the time delay between barrier and sample injection was $90 \mathrm{~s}$. Other time delays are indicated in Results and Discussion and in the Figure captions. The third injection valve was situated in front of the sample injector. It was equipped with a large loop of $1.5 \mathrm{~mL}$ and served for the qualitative checks of sample recovery ${ }^{34}$ by injections of neat $\mathrm{N}, \mathrm{N}$-dimethylformamide, DMF, which is a highly efficacious desorption promoting liquid, a desorli for numerous polar polymers retained within bare silica gel. Thus estimated sample recoveries in present systems were always higher than $90 \%$. The sample loop had volume of $50 \mu \mathrm{L}$. The injected polymer concentration ranged between 1 and $5 \mathrm{mg} \cdot \mathrm{mL}^{-1}$. The volume of barriers was $1,000 \mu \mathrm{L}$. Entire volumes of barrier, sample, and displacer loops were always injected.

In the LC LCD experiments with the THF/toluene mixed mobile phase and with the barriers consisting of the THF/ toluene mixtures or of the neat toluene, temperature of the ELSD evaporator was set at $80^{\circ} \mathrm{C}$ ("Method 1"). Temperature of ELSD evaporator was set at $180^{\circ} \mathrm{C}$ ("Method 6") when DMF displacer was used. Otherwise, the detector responded to the DMF zone. On the other hand, ELSD did not respond properly to TCP and PPO at evaporator temperature $180^{\circ} \mathrm{C}$.

The eluents employed in present work were mixtures of THF with toluene $70 / 30$ and $80 / 20 \mathrm{wt}$./wt. The mobile phases were desorlis for polymers under study (compare the part RESULTS AND DISCUSSION). This means that all samples eluted in the SEC mode when their solutions in eluent were injected without any barrier. The barriers contained increased concentration of toluene adsorli. The actual compositions of eluents and barriers are given in the part RESULTS AND DISCUSSION, as well as in the Figure captions.

\section{Important Notes}

Mobile phase and barrier compositions are given in the weight parts. When describing eluent and barrier mixtures, THF desorli is always mentioned first. For example, eluent designated 70/30 contains $70 \mathrm{wt}$. parts of THF. In all experiments, samples were dissolved and injected in eluent. The chromatograms were recorded from the time of sample (not barrier) injection. However, the overall timing of experiment started in the moment of the barrier introduction. In most experiments, the time delay between barrier and sample introduction was $90 \mathrm{~s}$. If different, the particular time delay is marked in seconds directly in Figures. 


\section{RESULTS AND DISCUSSION}

\section{Selections/Tests of Mobile Phases}

The LC LCD mobile phase has to promote the at least prevailingly exclusion controlled elution of all sample constituents. According to our previous studies the LC LCD sample recovery increases with the eluent strength. ${ }^{34}$ At the same time, the barrier efficacy and/or its size/volume has to be boosted if eluent possesses very high elution strength. Otherwise the barrier may permit the break-through of the adsorbing macromolecules. Increased barrier size may, however adversely affect the sample recovery. Moreover, barriers of large size consume large part of the mobile phase volume, which is available for the separation itself. Evidently, a compromise has to be sought.

The actual strength of mobile phase for the particular polymer on the given column packing can be estimated from the course of the dependence $\log M v s$. $V_{\mathrm{R}}$, where $M$ stands for molar mass of polymer and $V_{\mathrm{R}}$ for the corresponding retention volume. For a more exact evaluation, hydrodynamic volume of macromolecules ${ }^{45}$ should be used instead of their molar mass. However the plots with molar mass give sufficient information needed for the qualitative evaluation of mobile phase behavior/ suitability provided the eluent is equally or at least similarly good solvent for polymers under investigation. This condition is fulfilled with present systems. In order to obtain the unbiased information, samples have to be dissolved and injected in the mobile phase proper. If a neat adsorli or desorli were applied for sample dissolution, the retention volumes would pertain to different separation procedures, namely to LC under limiting conditions of desorption-like ${ }^{30}$ or to LC under limiting conditions of adsorption, ${ }^{46}$ respectively and the observed retention volumes could afford incorrect information.

As known, a decrease of $V_{\mathrm{R}}$ with increasing molar mass or hydrodynamic volume of a polymer indicates that the separation process is largely controlled by the changes of conformational entropy of macromolecules. This is typical for the SEC or SEC-like separation modes. Molar mass independent $V_{\mathrm{R}} \mathrm{S}$ are salient for LC CC, and eventually, retention volumes rise with $M$ in the liquid chromatographic systems, where the effect of enthalpic interactions prevails. The slight adsorption effects manifest themselves by the displacements of the $\log M v s . V_{\mathrm{R}}$ dependences to higher retention volumes though their overall courses may remain SEC-like. ${ }^{14}$

The dependences $\log M$ vs. $V_{\mathrm{R}}$ for PMMAs with different stereoregularity are depicted in Figure 1. Figure 1a shows the data obtained with the neat THF while Figure $1 \mathrm{~b}$ belongs to mixed eluent 70/30. For comparison, the curve obtained for PS standards is shown without the data points. It has been repeatedly confirmed that PS does not adsorb on bare silica gel from the neat THF or toluene eluents. The mutual position of the $\log M$ vs. $V_{\mathrm{R}}$ dependences for polymers of distinct stereoregularities can be affected by the effect tacticity on the hydrodynamic volume of macromolecules. The sizes of polymer chains of the same molar mass in solution depend on

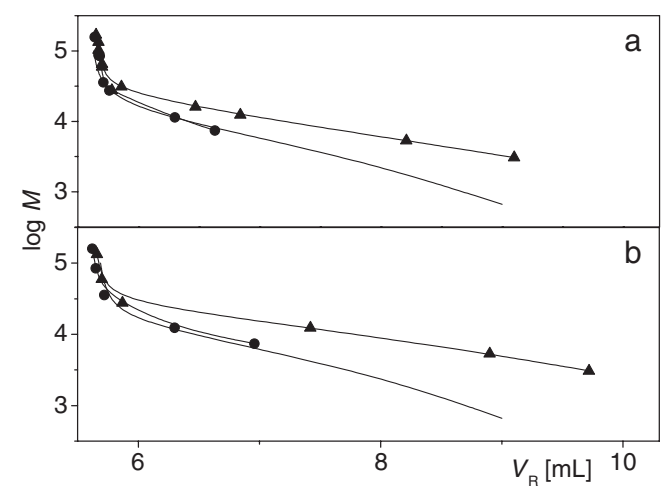

Figure 1. $\log M$ vs. $V_{\mathrm{R}}$ dependences for PS (lines without data points) and for st.-PMMA ( $\boldsymbol{\Delta})$ and it.-PMMA (O), respectively. Kromasil 60. Eluent: (a) neat THF; (b) THF/toluene 70/30.

their stereoregularity. ${ }^{47}$ This effect is rather small in the good solvents. ${ }^{16,17}$ Therefore, the large shift of $\log M$ vs. $V_{\mathrm{R}}$ dependence for any other polymer and any other eluent to higher retention volumes compared to PS/THF system mainly indicates presence of adsorption-provided the above described conditions related to the good thermodynamic quality of eluent toward polymer under study are fulfilled. The courses of curves in Figure 1a demonstrate that neat THF is a desorli of only medium efficacy for st.-PMMA and that this polymer is rather markedly retained by adsorption within the column packed by bare silica gel. The adsorption of it.-PMMA in the neat THF can be neglected. It should be noted that Chiantore ${ }^{48}$ has observed a rise of retention volume of PMMA (with unknown stereoregularity) as compared to PS in neat THF. The adsorption of st.-PMMAs in the eluent 70/30 is higher than in the neat THF. On the other hand, the adsorption effect is small for it.-PMMAs and it hardly exceeds the experimental errors. Similar tendency was observed with the mobile phase that contained $80 \mathrm{wt}$. $\%$ of THF (results not shown). The enhanced adsorption of st.-PMMA on bare silica gel compared with it.-species, however cannot be generalized. The extent of adsorption of it.-PMMA is larger on bare silica gel than that of syn.-PMMA if eluent is formed by a mixture of $\mathrm{CHCl}_{3}$ and ethanol. ${ }^{16}$

The above-described procedure was repeated with the samples of PEMA. The $\log M$ vs. $V_{\mathrm{R}}$ dependences for the PS standards, as well as for it.- and it.-PEMA are shown in Figure 2. Similar to PMMAs, syndiotactic poly(ethyl methacrylate)s are more adsorbed than the isotactic species in both neat THF and 70/30 eluent. However the effect of adsorption on retention volumes is rather small. The increased adsorption of st.-PEMA compared with it.-PEMA was also observed in LC $\mathrm{CC}$ with the columns packed with aminopropyl silica gel and the mixed eluents THF/cyclohexane. ${ }^{17}$

In the range of experimental errors, the retention volumes of excluded macromolecules are not affected by adsorption in the neat THF and in mixed eluent 70/30.

Similar results were obtained with mixed eluent $\mathrm{THF} /$ toluene $80 / 20$. It can be concluded that at $30^{\circ} \mathrm{C}$ the $\mathrm{THF} /$ toluene mobile phases $70 / 30$ and $80 / 20$ act as efficient desorlis 


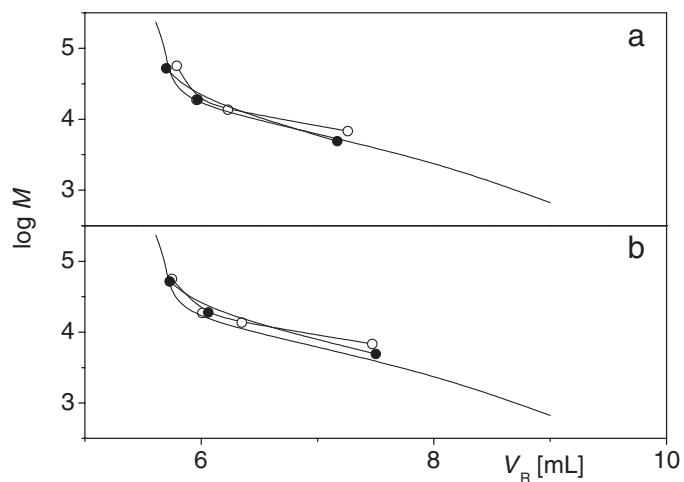

Figure 2. $\log M$ vs. $V_{\mathrm{R}}$ dependences for PS (lines without data points) and for st.-PEMA (O) and it.-PEMA (O), respectively. Kromasil 60. Eluent: (a) neat THF; (b) THF/toluene 70/30.

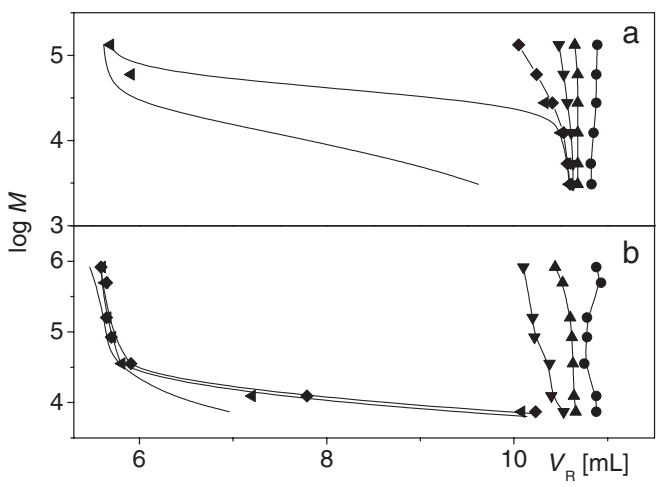

Figure 3. Effect of barrier composition. $\log M$ vs. $V_{\mathrm{R}}$ dependences for st.PMMA (a) and it.-PMMA (b); eluent 70/30; Kromasil 60. Lines without data points: no barrier applied. Barrier compositions: THF/

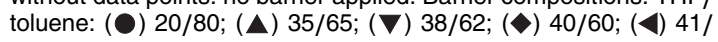
59.

for polymers under study and thus they can be used as eluents in the corresponding LC LCD measurements. Eluent THF/ toluene 70/30 of the medium desorption efficacy was used in most present experiments.

\section{LC LCD Measurements}

The effects of barrier composition on the retention of it.- and it.-PMMA are demonstrated in Figure 3. The results confirm that st.-PMMA is more strongly adsorbed than the it.-PMMA also under the LC LCD conditions. In comparison with it.-PMMA, less toluene in the barrier is needed to ensure its adsorli action and to decelerate elution of the st.-species. Up to a certain barrier composition the LC LCD system is well insensitive to the amount of adsorli in the barrier. This demonstrates large robustness and user-friendliness of the LC LCD method, which allows efficient separation of distinct polymers - as far as the difference between optimum barrier compositions for polymers to be separated is large enough. This is unfortunately not the case for it.- and st.-poly(methacrylate)s. Near certain barrier composition the LC LCD system starts to be sensitive to the barrier composition. Within about $1 \%$ of toluene content, the elution order flips over from the SEC to the LC LCD mode or vice versa. Notice the peculiar

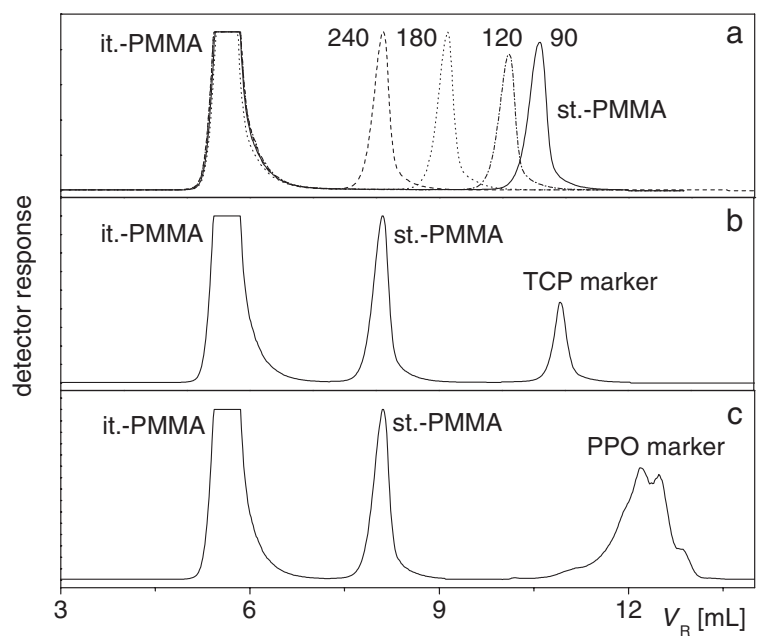

Figure 4. Chromatograms of mixtures of it.- and st.-PMMA with molar masses 27.5 and $28.6 \mathrm{~kg} \cdot \mathrm{mol}^{-1}$, and $\mathrm{c}_{\mathrm{i}}=5$ and $1 \mathrm{mg} \cdot \mathrm{mL}^{-1}$, respectively. Eluent 70/30; Kromasil 60. Barrier composition: 40/ 60 (a) effect of time delay between barrier and sample injection indicated in seconds; (b) time delay $240 \mathrm{~s}$, sample spiked with $0.1 \mathrm{mg} \cdot \mathrm{mL}^{-1}$ of tricresylphosphate; (c) time delay $240 \mathrm{~s}$, sample spiked with $0.3 \mathrm{mg} \cdot \mathrm{mL}^{-1}$ of PPG 400 .

behavior of st.-PMMA with a less efficacious barrier THF/ toluene $41 / 59$. It seems that at certain molar mass polymer breaks-through the barrier and, concerning its molar mass, elutes highly selectively. This behavior was also observed with some conventionally eluted polymer samples without any barrier and it was termed adsorption assisted SEC. ${ }^{49,50}$ Similar phenomenon is also observed for lower it.-PMMAs, which seem to be initially blocked by the barrier but later they breakthrough and elute in the SEC mode. The barrier THF/toluene $40 / 60$ already efficaciously decelerates fast elution of syn.-PMMA but it still allows the SEC or SEC-like elution of it.-PMMA with higher molar masses. This is the appropriate barrier composition, which would enable molar mass independent mutual separation of it.- and st.-PMMAs with higher molar masses. Unfortunately the above-mentioned aggregation between high molar mass it.-, and syn.-PMMA precluded majority of such separations. Most mixtures of it.-, and it.PMMAs with similar molar masses afforded ill shaped chromatograms. Their shape was irreproducible, it depended on the way the mixed solution was prepared, and it changed with time (results not shown). The exemption from the aggregation affected behavior is the couple of it.- and st.-PMMAs with the medium high molar masses 27.5 and $28.6 \mathrm{~kg} \cdot \mathrm{mol}^{-1}$, respectively, which could be separated into the two well defined peaks (Figure 4), fully identical with the peaks of separately eluted polymers. This result demonstrates that LC LCD enables separation of medium high PMMAs with unlike stereoregularity in spite of their practically identical molar masses. In Figure 4a the effect of time delay between injection of sample and barrier is also demonstrated. ${ }^{34}$ Sample injected later has more time to elute in the SEC mode before its deceleration by the barrier. As a consequence, the longer time delay between barrier and sample injection, the lower is the 


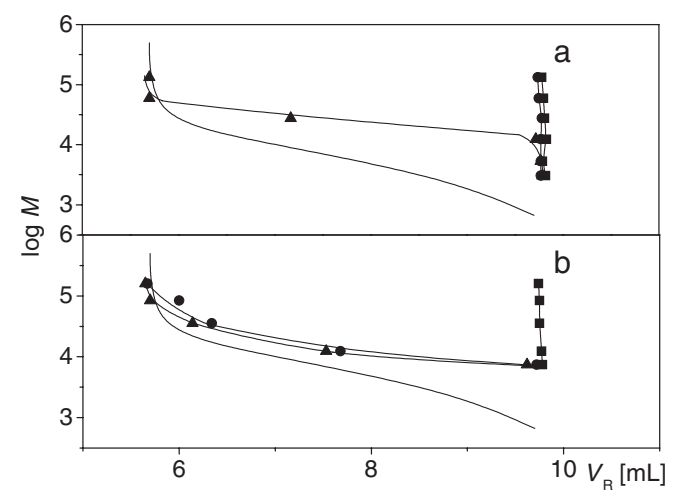

Figure 5. Effect of barrier composition. $\log M$ vs. $V_{\mathrm{R}}$ dependences for st.PMMA (a) and it.- (b) PMMA; eluent 70/30; Kromasil 100. Barrier compositions: ( $\square$ ) 38/62; (O) 40/60; ( $\boldsymbol{\Delta}$ ) 42/58. For comparison, the lines for PS without data points are shown.

retention volume of macromolecules, which are eventually blocked by barrier. Appropriate time delay between barrier and sample injection allows efficient separation of low molecular or oligomeric impurities/additives present in the sample and to provide enough space on the chromatograms to accommodate additional peaks without interference with the sample peaks (Figure $4 \mathrm{~b}$ and $4 \mathrm{c}$ ). ${ }^{34}$ Tricresylphosphate, TCP and poly(propylene oxide), PPO oligomer have been chosen as markers for impurities/additives. They elute well from the bare silica gel in mobile phases of medium polarity and due to their high boiling point they are easily detected by the ELS detector. The results furnish evidence that the LC LCD procedure can also be utilized for purification of some complex polymer systems. Notice the indication of the increased separation selectivity for poly(propylene oxide) oligomers according to their molar masses in present system (Figure 4c). ${ }^{34}$ The exclusion and adsorption has been combined to afford adsorption assisted SEC. ${ }^{49,50}$

The results obtained with silica gel with larger pores, $10 \mathrm{~nm}$ are depicted in Figure 5. As expected, the excluded polymer molar mass is higher for Kromasil 100 when compared with Kromasil 60 but otherwise, the results are similar. The optimum barrier composition is again THF/toluene 40/60.

Likewise, the barrier composition 40/60 rendered suitable in combination with eluent THF/toluene 80/20 (Figure 6). This again demonstrates that the barrier composition plays decisive role in the deceleration of adsorbing macromolecules in LC LCD. The conclusion is rather rational given the direct role of barrier in the deceleration of sample elution.

The effects of the barrier composition on elution of PEMAs are shown in Figure 7. Due to the lower adsorptivity of PEMA compared to PMMA, the barriers, which efficiently block fast elution of poly(ethyl methacrylate)s must contain more toluene. Suitable barriers for separation of it.-, from st.-PEMA on bare silica gel contain 17 to $18 \mathrm{wt}$ \% of toluene. Similar to PMMA the retention volumes are shifted toward higher values when the efficacy of barrier is increased that is when the content of adsorli toluene in barrier increases. This presumably results from the increased retention volume of barriers with

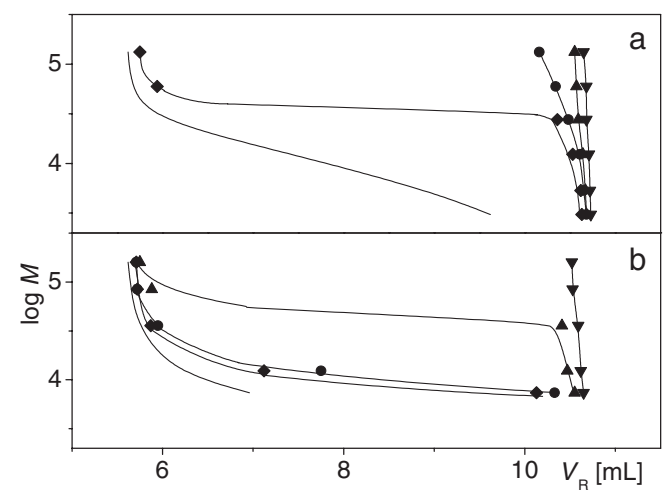

Figure 6. Effect of barrier composition. $\log M v s . V_{\mathrm{R}}$ dependences for st.- (a) and it.- (b) PMMA; eluent 80/20; Kromasil 60. Lines without data points: no barrier applied. Barrier compositions: ( $\boldsymbol{\nabla}$ ) 36/64; (A) 38/62; (○) 40/60; (४) 41/59.

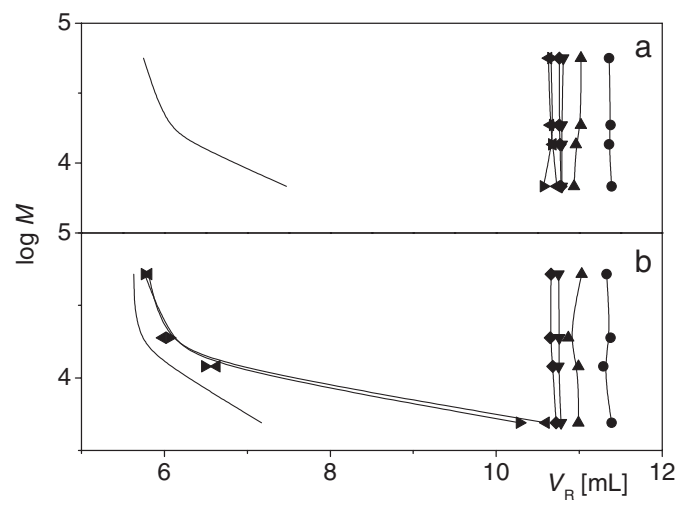

Figure 7. Effect of barrier composition. $\log M$ vs. $V_{\mathrm{R}}$ dependences for st.-PEMA (a) and it.-PEMA (b); eluent 70/30; Kromasil 60. Lines without data points: no barrier applied. Barrier compositions: (O) neat toluene; THF/toluene: $(\boldsymbol{A})$ 10/90; ( $\boldsymbol{\nabla}$ ) 15/85; ( $\boldsymbol{\Delta}$ ) 16/84; (ব) $17 / 83$; (>) 18/82.

their decreased strength/polarity. As known, such behavior forms a basis for high performance liquid chromatographic separation of low molar mass substances under normal phase conditions. In LC LCD, the adsorbed macromolecule elutes behind its barrier and therefore it leaves the column later when the elution volume of barrier rises. The answer to this question will be provided by detailed study of the role of barriers in the LC LCD, which is at present in preparation.

Some typical examples of separation of highly stereoregular PEMAs with similar molar masses are demonstrated in Figure 8. It is expected that by the application of shorter columns and/or increased elution rates ${ }^{34}$ the base-line discrimination of st.- and it.-PEMAs can be accomplished in less than five minutes, irrespective of their molar mass. However, such separations can only work for polymers that exhibit sufficiently high molar mass. Retention volumes of too small SEC eluted macromolecules approach those of barrier and they may become similar to the retention volume of the LC LCD retained species (Figure $8 \mathrm{~b}$ ).

Similar to st.-PMMA, the adjustment of time delay between barrier and sample introduction also enables optimization of 


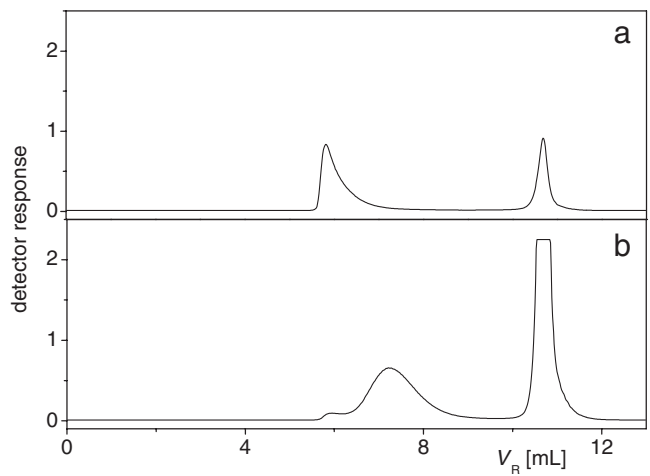

Figure 8. Chromatograms of mixtures of it.- and st.-PEMA with molar masses (a): 52.0 and $56.2 \mathrm{~kg} \cdot \mathrm{mol}^{-1}$, at $c_{1}=0.3$ and $0.5 \mathrm{mg} \cdot \mathrm{mL}^{-1}$, respectively; (b): 18.9 and $18.7 \mathrm{~kg} \cdot \mathrm{mol}^{-1}$, at $c_{1}=1$ and $3 \mathrm{mg} \cdot \mathrm{mL}^{-1}$, respectively. Eluent $70 / 30$; Kromasil 60 . Barrier composition: $17 / 83$.

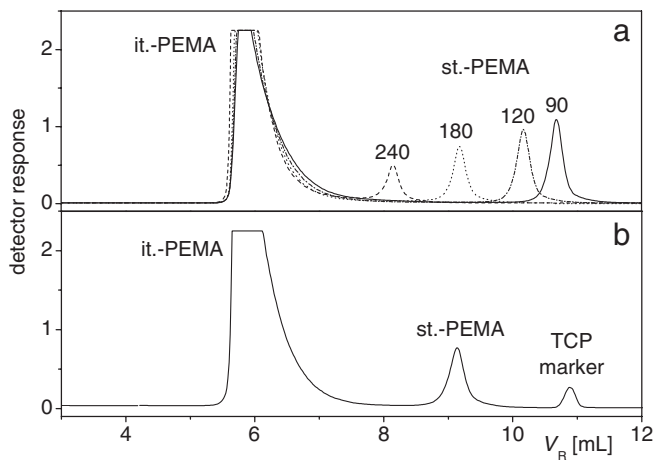

Figure 9. Chromatograms of mixture of it.- and st.-PEMA with molar masses 52.0 and $56.2 \mathrm{~kg} \cdot \mathrm{mol}^{-1}$, respectively. Each mixed polymer sample was prepared separately; $c$ for it.-PEMA between 3 and $5 \mathrm{mg} \cdot \mathrm{mL}^{-1}$ and for st.-PEMA between 0.3 and $0.7 \mathrm{mg} \cdot \mathrm{mL}^{-1}$. (a) Effect of time delay between barrier and sample injection. The time delays in seconds are introduced in Figure. (b) Separation of TCP marker from it.- and st.-PEMA samples. Time delay between barrier and sample injection was $180 \mathrm{~s}$.

retention volume of st.-PEMA (Figure 9a) and, consequently a comfortable separation of low molecular or oligomeric admixtures/impurities from the polymeric sample constituents. This is demonstrated in Figure 9b.

The difference in adsorptivity of it.-, and st.-poly(methacrylate)s in present chromatographic system is rather small and it likely results in the similarity in optimum barrier composition for both kinds of polymers. This feature would presumably prevent application of bare silica gel/THF/toluene system to an efficient LC LCD separation of common poly(methacrylate)s into series of fractions with narrow tacticity distribution by application of multiple barriers with a successively increased blocking efficacy. On the other hand, the results demonstrate the ability of LC LCD to mutually separate highly stereoregular poly(methacrylate)s irrespectively of their molar masses. Thus, LC LCD can be applied as the first step in the two-dimensional separation and comprehensive characterization of some complex polymer systems. ${ }^{36,37}$ The LC LCD column size can be easily increased and the method can serve for the effective preparative removal of unwanted kinds of macromolecules and low molecular additives from the polymer blends, that is for purification purposes.

\section{CONCLUSIONS}

Slight difference in adsorptivity of unlike macromolecules can be utilized for separations based on the barrier principle of liquid chromatography under limiting conditions of desorption, LC LCD. The mixtures of medium molar mass poly(methyl methacrylate)s and poly(ethyl methacrylate)s with the high degree of opposite stereoregularities can be efficiently discriminated irrespectively of molar mass of their constituents. The sensitivity of the method, however does not allow to efficiently separate samples with continuous, broad distribution of stereoregularity. The upper molar mass limit of LC LCD has not been established, ${ }^{31}$ while the lower molar mass limit of the method presumably depends on the pore size of the column. The sample recovery in the system bare silica gel with $6 \mathrm{~nm}$ pores/ $\mathrm{THF} /$ toluene has been always higher than $90 \%$, which is better than in many LC separations under critical conditions of adsorption.

Acknowledgment. The authors acknowledge financial support from the Slovak Grant Agencies VEGA (project 2/0171/ 09) and APVV (project 0592-07). Thanks also belong to EKA Chemicals, Gothenburg, Sweden for the gift of Kromasil 60 and Kromasil 100 silica gels, and especially to Mrs. J. Tarbajovská for her excellent technical assistance.

Received: March 19, 2009

Accepted: October 25, 2009

Published: December 15, 2009

\section{REFERENCES}

1. Y. A. Lipatov and L. M. Sergeeva, "Adsorption of Polymers," Wiley, New York, 1974.

2. M. Kawaguchi, T. Yamamoto, and T. Kato, J. Colloid Interface Sci., 241, 293 (2001).

3. G. J. Fleer, M. A. Cohen Stuart, J. M. H. M. Scheintjens, T. Cosgrove, and B. Vincent, "Polymer at Interfaces," Chapman and Hall, London, 1993.

4. S. Teramachi, A. Hasegawa, Y. Shima, M. Akatsuka, and M. Nakajima, Macromolecules, 12, 992 (1979).

5. G. Glöckner, "Gradient HPLC of copolymers and chromatographic cross-fractionation," Springer, Berlin, 1991.

6. T. Mourey, J. Chromatogr., 357, 101 (1986).

7. a) H. Sato, M. Sasaki, and K. Ogino, Polym. J., 21, 965 (1989). b) H. Sato, M. Sasaki, and K. Ogino, Polym. J., 23, 23 (1991).

8. T. Schunk, J. Chromatogr., A, 656, 591 (1993).

9. S. Mori, Macromol. Symp., 110, 87 (1996).

10. B. G. Belenkii, E. S. Gankina, M. B. Tennikov, and L. Z. Vilenchik, Dokl. Akad. Nauk, 231, 1147 (1976).

11. B. G. Belenky, M. D. Valchikhina, I. A. Vakhtina, E. S. Gankina, and O. G. Tarakanov, J. Chromatogr., A, 129, 129 (1976).

12. B. G. Belenky, E. S. Gankina, M. B. Tennikov, and L. Z. Vilenchik, J. Chromatogr., A, 147, 99 (1978).

13. H. Pasch and B. Trathnigg, "HPLC of Polymers," Springer, Berlin, 1998.

14. D. Berek, Prog. Polym. Sci., 25, 873 (2000).

15. D. Berek, M. Janco, T. Kitayama, and K. Hatada, Polym. Bull., 32, 
629 (1994).

16. D. Berek, M. Janco, K. Hatada, T. Kitayama, and N. Fujimoto, Polym. J., 29, 1029 (1997).

17. M. Janco, T. Hirano, T. Kitayama, K. Hatada, and D. Berek, Macromolecules, 33, 1710 (2000).

18. T. Kitayama, M. Janco, K. Ute, R. Niimi, K. Hatada, and D. Berek, Anal. Chem., 72, 1518 (2000).

19. H. J. A. Philipsen, B. Klumperman, and A. L. German, J. Chromatogr., A, 746, 211 (1996).

20. D. Berek, Macromol. Symp., 110, 33 (1996).

21. D. Berek, M. Janco, and G. Meira, J. Polym. Sci., Part A: Polym. Chem., 36, 1363 (1998).

22. D. Berek, Macromolecules, 37, 6096 (2004).

23. D. Berek and A. Russ, Chem. Pap.-Chem. Zvesti, 60, 249 (2006).

24. E. Beaudoin, A. Favier, C. Galindo, A. Lapp, C. Petit, D. Gigmes, S. Marque, and D. Bertin, Eur. Polym. J., 44, 514 (2008).

25. A. Favier, C. Petit, E. Beaudoin, and D. Bertin, e-Polymer, 009, 1 (2009).

26. S. Teramachi, A. Hasegawa, and T. Matsumoto, J. Chromatogr., 547, 429 (1991).

27. D. Cho, S. Park, T. Chang, K. Ute, I. Fukuda, and T. Kitayama, Anal. Chem., 74, 1928 (2002).

28. H. C. Lee and T. Chang, Polymer, 37, 5747 (1996).

29. T. Chang, H. C. Lee, W. Park, and C. Ko, Macromol. Chem. Phys., 200, 2188 (1999).

30. D. Berek, Macromolecules, 31, 8517 (1998).

31. M. Snauko and D. Berek, J. Chromatogr., A, 1094, 42 (2005).

32. D. Berek, Eur. Polym. J., 45, 1798 (2009).

33. D. Berek, Macromol. Chem. Phys., 209, 695 (2008).

34. D. Berek, Macromol. Chem. Phys., 209, 2213 (2008).
35. M. Snauko and D. Berek, Chromatographia, 57, S-55 (2003).

36. D. Berek, Macromol. Symp., 174, 413 (2001).

37. D. Berek, in "Handbook of Size Exclusion Chromatography and Related Techniques” C.-S. Wu, Ed., Marcel Dekker, New York, 2004.

38. K. Hatada, K. Ute, K. Tanaka, T. Kitayama, and Y. Okamoto, Polym. J., 17, 977 (1985).

39. K. Hatada, K. Ute, K. Tanaka, Y. Okamoto, and T. Kitayama, Polym. J., 18, 1037 (1986)

40. K. Hatada, K. Ute, T. Kitayama, M. Yamamoto, T. Nishimura, and M. Kashiyama, Polym. Bull., 21, 489 (1989).

41. T. Kitayama, T. Shinozaki, T. Sakamoto, M. Yamamoto, and K Hatada, Makromol. Chem. Suppl., 15, 167 (1989).

42. T. Kitayama, N. Fujimoto, T. Yanagida, and K. Hatada, unpublished results.

43. T. Kitayama, T. Shinozaki, E. Masuda, M. Yamamoto, and K Hatada, Polym. Bull., 20, 505 (1988).

44. M. Kurata and Y. Tsunashima, in "Polymer Handbook," IVth ed., J. Brandrup, E. H. Immergut, E. A. Gruelke, A. Abe, and D. R. Bloch, Ed., Wiley, New York, 1999, Chapter VII

45. Z. Grubisic, P. Rempp, and H. Benoit, J. Polym. Sci., Part B: Polym. Phys., 5, 573 (1967).

46. D. Berek and D. Hunkeler, J. Liq. Chromatogr. Related Technol., 22, 2867 (1999).

47. H. Yamakawa, "Helical Wormlike Chains in Polymer Solutions," Springer, Berlin, 1997.

48. O. Chiantore, J. Liq. Chromatogr., 7, 1 (1994).

49. D. Berek and D. Bakos, J. Chromatogr., 91, 237 (1974).

50. A. Russ and D. Berek, J. Sep. Sci., 30, 1852 (2007). 